\title{
A precise and accurate distance to the Large Magellanic Cloud from late-type eclipsing-binary systems
}

\author{
G. Pietrzyński, ${ }^{1,2}$ W. Gieren, ${ }^{1}$ D. Graczyk,${ }^{1}$ I. Thompson, ${ }^{3}$ \\ B. Pilecki, ${ }^{1,2}$ N. Nardetto,${ }^{8}$ R.-P. Kudritzki, ${ }^{9}$ F. Bresolin, ${ }^{9}$ G. Bono, ${ }^{4,5}$ \\ P. Prada Moroni, ${ }^{6,7}$ P. Konorski, ${ }^{2}$ M. Gorski, ${ }^{2}$ J. Storm,,${ }^{10}$ \\ R. Smolec, ${ }^{11}$ and P. Karczmarek ${ }^{2}$ \\ ${ }^{1}$ Universidad de Concepción, Departamento de Astronomía, Casilla 160-C, Concepción, Chile \\ ${ }^{2}$ Warsaw University Observatory, Aleje Ujazdowskie 4, 00-478 Warszawa, Poland \\ ${ }^{3}$ Carnegie Observatories, 813 Santa Barbara Street, Pasadena, CA 91101-1292, USA \\ ${ }^{4}$ Dipartimento di Fisica Università di Roma Tor Vergata, via della Ricerca Scientifica 1, \\ 00133 Rome, Italy \\ ${ }^{5}$ INAF-Osservatorio Astronomico di Roma, Via Frascati 33, 00040 Monte Porzio Catone, Italy \\ ${ }^{6}$ Dipartimento di Fisica Università di Pisa, Largo B. Pontecorvo 2, 56127 Pisa, Italy \\ ${ }^{7}$ INFN, Sez. Pisa, via E. Fermi 2, 56127 Pisa, Italy \\ ${ }^{8}$ Laboratoire Fizeau, UNS/OCA/CNRS UMR6525, Parc Valrose, 06108 Nice Cedex 2, France \\ ${ }^{9}$ Institute for Astronomy, 2680 Woodlawn Drive, Honolulu, HI 96822, USA \\ ${ }^{10}$ Leibniz Institute for Astrophysics, An der Sternwarte 16, 14482, Potsdam, Germany \\ ${ }^{11}$ Nicolaus Copernicus Astronomical Centre, Bartycka 18, 00-716 Warszawa, Poland
}

\begin{abstract}
We present a precise and accurate measurement of the distance to the Large Magellanic Cloud based on late-type eclipsing-binary systems. Our results provide curently the most accurate zero point for the extragalactic distance scale.
\end{abstract}

Keywords. binaries: eclipsing, Magellanic Clouds, galaxies: individual (LMC), distance scale

\section{Introduction}

Detached eclipsing double-lined spectroscopic binaries offer a unique opportunity to measure directly, and very accurately, stellar parameters like mass, luminosity, and radius (Andersen 1991), as well as distance (Paczyński 1997; for a very detailed historical review, see also Kruszewski \& Semeniuk 2000). It has been argued (Paczyński 2000) that with current observational facilities, and upon application of an appropriate surfacebrightness-color relation, eclipsing binaries have the potential to yield the most direct (one-step) and most accurate (approximately 2\%) distance to the Large Magellanic Cloud (LMC). Detailed descriptions of this approach can be found in Paczyński (1997) and Kruszewski \& Semeniuk (2000). Briefly, using high-quality spectroscopic and photometric observations, standard fitting routines (e.g., Wilson \& Devinney 1971; Wilson 1990) provide very accurate masses, sizes, and surface-brightness ratios for the components of a double-lined eclipsing binary (e.g., Andersen 1991). The distance to an eclipsing binary follows from the dimensions determined this way, combined with the absolute surface brightness, which can be inferred from the observed spectral types on the basis of either theoretical or empirical calibrations. An empirical surface-brightness-color relation is already very well-established for stars with spectral types later than A5, based 


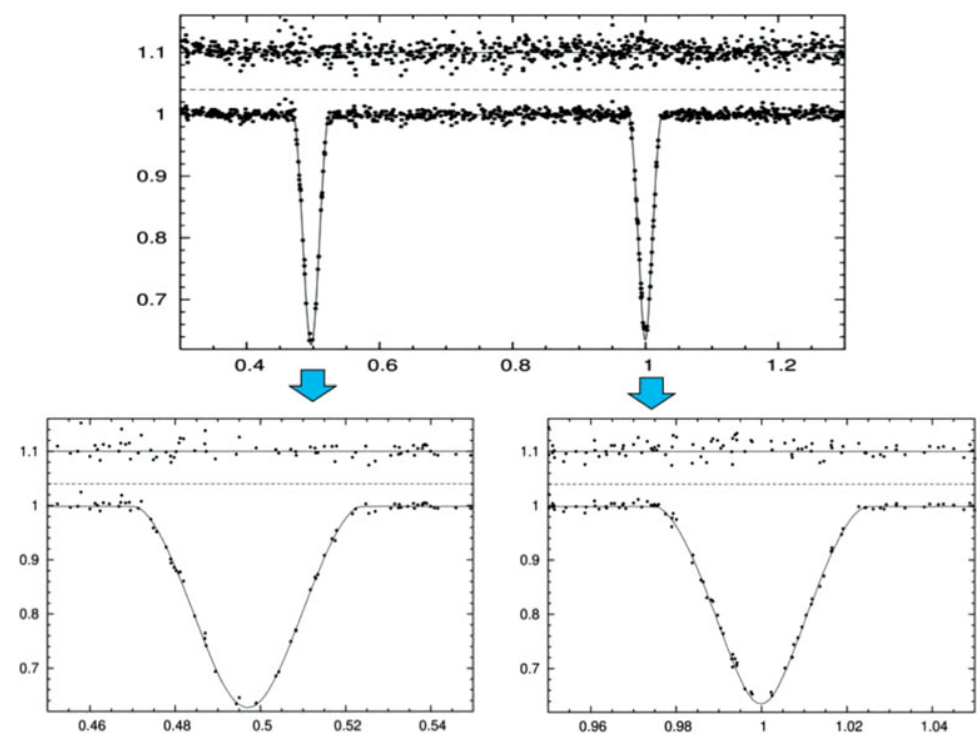

Figure 1. Observed $I$-band light curve and photometric solution, obtained from analysis based on the Wilson-Devinney code for the system OGLE-051019.64-685812.3

on accurate determinations of stellar angular diameters using interferometry (Benedetto 1998; Groenewegen 2004; Kervella et al. 2004; Di Benedetto 2005). It is nearly parallel to the reddening line for late-type stars (Barnes \& Evans 1976). The only concern associated with using this approach is that late-type main-sequence binaries located in the LMC are too faint to secure accurate, high-resolution spectra even with the largest telescopes. For that reason, for a long time only the early-type systems had been used for distance determination to the LMC (e.g., Fitzpatrick et al. 2003; and references therein). However, a well-calibrated surface-brightness-color relation is still not available for such stars, so they were forced to use the so-called 'classical' approach, including the need to use large and uncertain bolometric corrections and requiring a multidimensional fit of many different parameters at the same time (see Fitzpatrick et al. 2003).

\section{Late-type systems}

The situation changed when our group obtained photometric solutions using the Wilson-Devinney code for eclipsing binaries in the LMC from the OGLE catalogs and discovered a dozen of G-type systems, ideal for distance determination. For these latetype systems an accurate surface-brightness- $(V-K)$ color calibration is available, and by securing several $K$-band observations outside the eclipses, combined with measuring their orbital radial-velocity-curve amplitudes from high-resolution spectroscopy (our objects are bright enough at $V=16.5 \mathrm{mag}$ ), the full potential of eclipsing binaries as distance indicators can be exploited for the first time for these late-type systems.

We have obtained high-resolution spectroscopy with HARPS at the $3.6 \mathrm{~m}$ telescope and with MIKE at the Magellan Clay telescope, as well as $K$-band observations outside the eclipses with SOFI at the NTT for our systems over the last 10 years. For the system designated OGLE-LMC-SC10-1838, the results of our distance analysis have recently been published (Pietrzyński et al. 2009; see also Figs 1 and 2). By applying the accurate surface-brightness-color calibration of Di Benedetto (2005), we measured the distance to this eclipsing binary as $50.2 \pm 1.3 \mathrm{kpc}$. The accuracy of our distance determination 


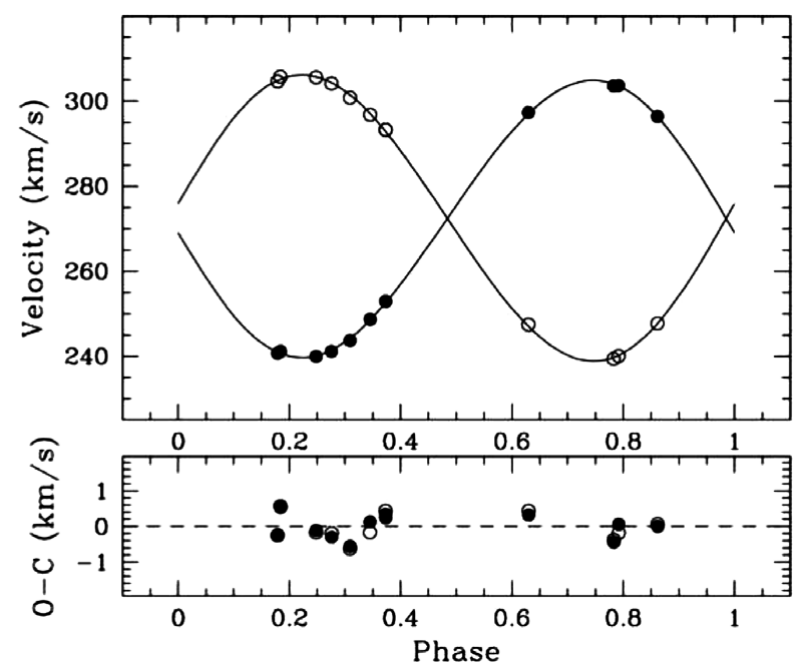

Figure 2. Spectroscopic orbit of one of our late-type binaries, OGLE-051019.64-685812.3

was affected by the accuracy of the radial-velocity-curve amplitude $(0.5 \%)$, the relative radii $(1 \%)$, the zero point of the optical $(0.6 \%)$ and near-infrared $(0.8 \%)$ photometry, the reddening ( $1 \%$; this small uncertainty is only owing to our analysis using $K$-band data), and the calibration of the surface-brightness-color relation (2\%). Currently, we have collected all necessary data for eight systems. The quality of the data is very similar to that shown in Figs 1 and 2. The preliminary LMC distance determinations based on all these systems is $d_{\mathrm{LMC}}=49.96 \pm 0.19$ (statistical) \pm 1.11 (systematic) kpc.

\section{Conclusions}

Our $2 \%$ direct measurement of the LMC distance provides the currently most accurate zero point for the extragalactic distance scale. Moreover, since the LMC contains a large variety of different distance indicators, our results provide an excellent way to calibrate the zero points of these different techniques and thereby significantly improve the local distance scale. Once the calibration of the surface-brightness-color relation has been further improved, our technique has the potential to deliver a $1 \%$ LMC distance and form a firm basis for the determination of the Hubble constant with a precision of $2 \%$. Our group is also involved in studies of similar systems in the Small Magellanic Cloud (Graczyk et al. 2012). Having accurate and direct distances to both Magellanic Clouds, we will be able to check the effects of metallicity on the brightness of Cepheids and other distance indicators.

\section{References}

Andersen, J. 1991, A\&ARv, 3, 9

Barnes, T. G., \& Evans, D. S., 1976 MNRAS, 174, 489

Di Bededetto, G. P. 1998, A $\mathscr{E} A, 339,858$

Di Benedetto, G. P. 2005, MNRAS, 357, 174

Fitzpatrick, E. L., Ribas, I., Guinan, E. F., Maloney, F. P., \& Claret, A. 2003, ApJ, 587, 685

Gieren, W., Pietrzyński, G., Bresolin, F., et al. 2005, The ESO Messenger, 121, 23

Graczyk, D., Pietrzyski, G., Thompson, I. B., et al. 2012, ApJ, 750, 144

Groenewegen, M. A. T. 2004, MNRAS, 353, 903 
Kervella, P., Thévenin, F., Di Folco, E., \& Sgransan, D. 2004, A\&A, 426, 297

Kruszewski, A. \& Semeniuk, I. 2000, Acta Astron., 49, 561

Paczyński, B. 1997, in: The Extragalactic Distance Scale (Livio, M., ed.) STScI Symp. Ser., p. 273 (astroph/9608094)

Paczyński, B. 2000, A\&AS, 196, 1001

Pietrzyński, G., Thompson, I. B., Graczyk, D., et al. 2009, ApJ, 697, 862

Wilson, R. E. \& Devinney, E. J. 1971, ApJ, 166, 606

Wilson, R. E. 1990, ApJ, 234, 1054960894 